\title{
Efficacy and Safety of Regorafenib With 2/1 Schedule for Patients $\geq 75$ Years With Metastatic Colorectal Cancer (mCRC) After Failure of 2 Lines of Chemotherapy
}

\author{
Roberto Petrioli, ${ }^{1}$ Martina Chirra, ${ }^{1}$ Luciana Messuti, ${ }^{1}$ Anna Ida Fiaschi, ${ }^{2}$ \\ Vinno Savelli, ${ }^{3}$ Ignazio Martellucci, ${ }^{1}$ Edoardo Francini ${ }^{4}$
}

\begin{abstract}
Regorafenib was shown to improve survival of patients with metastatic colorectal cancer resistant or unfit for all available therapies. Data on the efficacy and safety of regorafenib in elderly patients are scarce. In this small analysis, regorafenib administered with a modified schedule ( 2 weeks-on/1 week-off) to patients who were aged $\geq \mathbf{7 5}$ and non-frail with late-stage metastatic colorectal cancer appears to be tolerable and effective. Background: In the CORRECT (patients with metastatic COloRectal Cancer treated with REgorafenib or plaCebo after failure of standard Therapy) trial, regorafenib was proven to extend survival of patients with metastatic colorectal cancer ( $m C R C$ ) that progressed after all available therapies. Grade 3 to 4 toxicity occurred in $54 \%$ of patients, and data on the activity and tolerability of regorafenib in elderly patients were scarce. The aim of this study was to evaluate the efficacy and safety of an alternative schedule, 2-week-on treatment and 1 week-off (2/1 schedule), of regorafenib for elderly patients with mCRC. Patients and Methods: Patients $\geq 75$ years with $\mathrm{mCRC}$ who progressed after oxaliplatin- and irinotecan-based chemotherapy received regorafenib on a $2 / 1$ schedule. Potentially frail subjects were identified by G8 screening tool and excluded. The 2-month disease-control rate was the primary endpoint, and the secondary endpoints included safety, progression-free survival (PFS), overall survival (OS), and objective response rate. Results: Between February 2014 and May 2017, 23 patients with mCRC were recruited at our institution. No partial or complete responses were observed, and the stable disease and disease-control rate were $52.2 \%$. The median PFS was 4.8 months (95\% confidence interval, 3.8-6.3 months), and the median OS was 8.9 months (95\% confidence interval, 6.9-10.6 months). Adverse events were uncommon, and the most frequent grade 3 toxicity adverse events were hand-foot skin reaction (9\%) and fatigue (9\%). Toxicity-related dose reductions and discontinuations occurred in 5 and 2 patients, respectively. Conclusion: Regorafenib administered with a modified 2/1 schedule to patients who were aged $\geq 75$ years and non-frail with treatment-refractory $\mathrm{mCRC}$ seems to be tolerable and achieve encouraging results in terms of PFS and OS.
\end{abstract}

Clinical Colorectal Cancer, Vol. m, No. - m-- @ 2018 Published by Elsevier Inc.

Keywords: Colorectal cancer, Elderly, Geriatric assessment, Metastatic cancer, Regorafenib

\footnotetext{
${ }^{1}$ Medical Oncology Unit, Department of Medicine, Surgery and Neurosciences, University of Siena, Siena, Italy

${ }^{2}$ Pharmacology Unit, Department of Medicine, University of Siena, Siena, Italy ${ }^{3}$ Department of Surgery and Bioengineering, Section of Surgery, University of Siena, Siena, Italy

${ }^{4}$ La Sapienza University, Rome, Italy
}

Submitted: Nov 25, 2017; Revised: Feb 12, 2018; Accepted: Feb 14, 2018

Address for correspondence: Roberto Petrioli, MD, Medical Oncology Unit, Department of Medicine, Surgery and Neurosciences, University of Siena, Viale Bracci, Siena 53100, Italy

E-mail contact: r.petrioli@ao-siena.toscana.it

\section{Introduction}

Colorectal cancer (CRC) is one of the most frequent malignancies worldwide. It is more common in the elderly ( $\geq 65$ years), with approximately $60 \%$ of diagnoses in patients aged 65 years or over. ${ }^{1}$ The average life expectancy in the developed world is rapidly increasing and so is the incidence of bowel cancer among elderly patients. However, this category is still underrepresented in clinical trials, and data supporting treatment for elderly patients with advanced CRC is scarce. ${ }^{2,3}$

The treatment of mCRC has drastically changed with the advent of targeted therapies. These molecules include regorafenib, an oral 


\section{Regorafenib in Elderly Patients}

multikinase inhibitor that targets the multiple proangiogenic signaling pathways inhibiting vascular endothelial growth factor receptor (VEGF-R), fibroblast growth factor receptor (FGF-R), and platelet-derived growth factor receptor (PDGF-R) and targets other signaling oncogenic pathways such as KIT, RET, RAF-1, and BRAF, and immunoglobulin and EGF tyrosine kinase. ${ }^{4,5}$ In the CORRECT (patients with metastatic COloRectal Cancer treated with REgorafenib or plaCebo after failure of standard Therapy) randomized trial, regorafenib was shown to improve overall survival (OS) of patients with mCRC previously treated with fluoropyrimidine-, oxaliplatin-, and irinotecan-based chemotherapy, or antiVEGF or, in KRAS wild-type, anti-epidermal growth factor receptor (EGFR) targeted therapy compared with placebo (6.4 vs. 5.0 months; hazard ratio $[\mathrm{HR}], 0.77 ; 95 \%$ confidence interval [CI], 0.64-0.94; $P=.0052) .{ }^{6}$ Although the subgroup analysis reported that patients $\geq 65$ years achieved a survival benefit (HR, 0.86; $95 \%$ CI, 0.61-1.19), the information for patients $\geq 75$ years is limited owing to their small number in this group (in the CORRECT trial, only 38 patients $\geq 74$ years received regorafenib).

Additionally, toxicity was notably more severe with regorafenib than placebo, with grade 3 or 4 treatment-related adverse events occurring in $54 \%(\mathrm{~N}=270)$ of the regorafenib-treated patients versus $14 \%(\mathrm{~N}=35)$ of patients receiving placebo. Owing to the adverse events, dose reductions were observed in $38 \%(\mathrm{~N}=188)$ of patients, and therapy was interrupted in $61 \%(\mathrm{~N}=304)$ of patients. Additionally, it should be noted that the median age of the population was only 61 years, and adverse events data specific to the elderly population were not reported. ${ }^{6}$ Therefore, evidence supporting use of regorafenib in elderly patients is currently weak. However, older adults with mCRC who failed the guidelinesrecommended chemotherapy regimens and still have a good performance status (PS) and are considered fit on a comprehensive geriatric assessment (CGA) are not rare and might benefit from an active antitumoral treatment. ${ }^{7,8}$ In this regard, regorafenib could be a viable option as last line of treatment if proven efficient and safe.

The standard dosing schedule for regorafenib is $160 \mathrm{mg}$ once daily for 3 consecutive weeks followed by 1 week off ( $3 / 1$ schedule), and most of the severe side effects occurred during early phases of exposure (after 1-2 cycles). To our knowledge, data in the literature concerning alternative schedules for this drug or the safety and efficacy of regorafenib in older adults are limited. With the aim to improve the toxicity profile of regorafenib, a modified schedule ( 2 weeks on treatment followed by 1 week off), entailing a shorter exposure to the drug, was tested on a small cohort of elderly patients at our institution. Based on the achieved promising preliminary findings (unpublished data), the current study was designed to evaluate the activity and safety of an alternative $2 / 1$ schedule of regorafenib in patients who were age $\geq 75$ years with $\mathrm{mCRC}$ who progressed after 2 or more previous chemotherapy lines.

\section{Patients and Methods Eligibility Criteria}

Patients $\geq 75$ years with documented mCRC who had progressed on previous oxaliplatin- and irinotecan-based chemotherapy were enrolled at our institution in this prospective observational study. The other eligibility criteria included age of 18 years or greater, Eastern Cooperative Oncology Group (ECOG) PS of 0 to 2 , bidimensionally measurable disease, a life expectancy of at least 3 months, adequate hematologic parameters (an absolute neutrophil count of $\geq 1.5 \times 10^{9} / \mathrm{L}$ and a platelet count of $\geq 100 \times 10^{9} / \mathrm{L}$ ), creatinine serum levels less than 1.5 times the upper limit of the normal range, and total bilirubin levels less than 3-fold the upper normal limit; aspartate and alanine aminotransferase less than 3-fold the upper normal limit, and absence of a second primary tumor other than non-melanoma skin cancer or in situ cervical carcinoma, at baseline. Exclusion criteria were brain metastases or prior treatment for brain metastasis; uncontrolled pleural or pericardial effusion; clinically significant cardiovascular disease; and medically uncontrolled hypertension.

At baseline, the G8 screening tool was used to identify potentially frail subjects among the recruited patients ${ }^{9}$; subjects with $\leq 14$ points were further evaluated by CGA. ${ }^{8}$ Patients classified as frail were excluded from the study. The baseline geriatric assessment included the Charlson Comorbidity Index and was performed by 2 medical oncologists (R.P. and L.M.) and a geriatrist (I.M.). ${ }^{10,11}$ Vulnerable patients were defined as subjects who resulted not independent in 1 or more activities according to the Instrumental Activities of Daily Living and had 1 or 2 comorbidities with intermediate comorbidity score.

All patients gave their written informed consent prior to starting treatment.

\section{Patient Evaluation}

A complete physical examination, monitoring of symptoms and toxic effects, assessment of renal function, and a complete blood count were performed on patients at day 1 of every cycle. In order to minimize the risk of administering a potentially toxic drug to very elderly patients without a clinical benefit, the disease was reassessed after completion of 3 2/1-cycles of regorafenib (approximately after 8 weeks), then every 2 months for 6 months, and thereafter at 3 -month intervals until there was evidence of disease progression. Objective tumor response was evaluated radiologically according to Response Evaluation Criteria in Solid Tumors (RECIST criteria, version 1.1).

\section{Treatment Delivery}

Patients received regorafenib $160 \mathrm{mg}$ once daily for 2 consecutive weeks of each 3 -week cycle ( $2 / 1$ schedule). The starting dose was reduced to $120 \mathrm{mg}$ in patients considered vulnerable or with $>1$ comorbidity and $80 \mathrm{mg}$ in patients $\geq 80$ years old or with an ECOG PS of 2.

The dose was re-escalated to a maximum of $160 \mathrm{mg} /$ day if no grade $\geq 2$ toxicity occurred.

\section{Toxicity}

The common toxicity criteria of the National Cancer Institute Common Terminology Criteria for Adverse Events (NCI-CTCAE; version 4.02) were used to assess toxicity. Treatment was delayed if, on the planned treatment day, the neutrophil count was $<1500 /$ $\mathrm{mm}^{3}$, the platelet count was $<100,000 / \mathrm{mm}^{3}$, or the patient had persistent diarrhea or stomatitis of grade 1 or higher. Any patient who required more than 3 weeks for recovery from adverse reactions was excluded from the study. In the event of grade 3 or greater 
Table 1 Patient Characteristics

\begin{tabular}{|c|c|}
\hline Characteristics & Patients, $N=23(\%)$ \\
\hline \multicolumn{2}{|l|}{ Age, y } \\
\hline Median & 78 \\
\hline Range & $75-87$ \\
\hline$\geq 80 \mathrm{y}$ & $8(35)$ \\
\hline \multicolumn{2}{|l|}{ Gender } \\
\hline Male & $16(70)$ \\
\hline Female & $7(30)$ \\
\hline \multicolumn{2}{|l|}{ ECOG PS } \\
\hline 0 & $6(26)$ \\
\hline 1 & $14(61)$ \\
\hline 2 & $3(13)$ \\
\hline \multicolumn{2}{|c|}{ Comprehensive geriatric assessment } \\
\hline Fit & $18(78)$ \\
\hline Vulnerable & $5(22)$ \\
\hline \multicolumn{2}{|c|}{ Charlson Comorbidity Index } \\
\hline Median (range) & $1(0-2)$ \\
\hline \multicolumn{2}{|l|}{ Primary tumor } \\
\hline Colon & $17(74)$ \\
\hline Rectum & $6(26)$ \\
\hline \multicolumn{2}{|l|}{ Metastatic sites } \\
\hline Liver & $18(78)$ \\
\hline Lymph nodes & $9(39)$ \\
\hline Peritoneum & $6(26)$ \\
\hline Lung & $8(35)$ \\
\hline Other & $3(23)$ \\
\hline Metastatic sites >1 & $16(70)$ \\
\hline \multicolumn{2}{|c|}{ Previous anti-cancer treatments } \\
\hline Oxaliplatin-based & $23(100)$ \\
\hline Irinotecan-based & $23(100)$ \\
\hline Anti-EGFR & $8(35)$ \\
\hline Anti-VEGF & $9(39)$ \\
\hline
\end{tabular}

Abbreviations: ECOG PS = Eastern Cooperative Oncology Group performance status; EGFR = epidermal growth factor receptor; VEGF = vascular endothelial growth factor.

hematologic or any other severe ( $\geq$ grade 3 ) organ toxicity, treatment was delayed, and at recovery, regorafenib doses were reduced by $40 \mathrm{mg}$ (to a minimum of $80 \mathrm{mg}$ ) daily for subsequent courses.

\section{Statistical Considerations}

The primary endpoint of the study was 2-month disease-control rate (DCR), defined as the percentage of patients who achieved stable disease (SD) or partial (PR) or complete response (CR) within 2 months after start of therapy.

Regorafenib was recently approved by the United States Food and Drug Administration and the European Medicine Agency as salvage treatment for patients with mCRC who progressed after, or are not considered fit for, available treatments including fluoropyrimidine-, oxaliplatin-, and irinotecan-based chemotherapy, or anti-VEGF therapy or, if KRAS wild type, anti-EGFR targeted therapy. The CORRECT study showed a 2-month DCR of approximately $41 \%$ for patients treated with regorafenib. Given that specific data was not reported for elderly patients, and assuming a $0 \%$ to $5 \% 2$-month DCR with best supportive care alone, the hypothesis for the current study was that using a modified schedule of regorafenib in patients $\geq 75$ years, at least $25 \%$ of subjects would be progression-free after 2 months from the start of treatment. It was calculated that a total of 21 patients should be recruited to yield a $80 \%$ probability to correctly select the treatment when it is superior by absolute difference of $20 \%$ in 2-month DCR (Simon minimax design). ${ }^{12}$

Secondary endpoints included safety, progression-free survival (PFS), defined as time from treatment start to disease progression or death from any cause, OS, as time elapsed from treatment initiation to death from any cause, and objective response rate, as the proportion of patients who achieved PR or CR. The Kaplan-Meier method was used to determine PFS and OS. Statistical analyses were conducted by STATA software.

\section{Results \\ Patient Characteristics}

Between February 2014 and May 2017, 23 patients > 75 years of age with mCRC were enrolled in the study. The baseline characteristics of patients are summarized in Table 1 . The median age was 78 years (range, $75-87$ years), and $35 \%(\mathrm{~N}=8)$ of patients were at least 80 years old. Most patients had an ECOG PS $\geq 1$ $(\mathrm{N}=17 ; 73.9 \%)$ and were considered fit by CGA $(\mathrm{N}=18 ; 78 \%)$, whereas $5(22 \%)$ were classified as vulnerable. The median G8 score of enrolled patients was 15 (range, 15-17) at baseline. The primary tumor was located in the colon in $17(74 \%)$ and the rectum in 6 (26\%) patients. Eighteen subjects (78\%) had liver metastases, and $17(74 \%)$ had at least 1 metastatic site. All patients had at least 1 comorbidity, and the majority $(\mathrm{N}=17 ; 74 \%)$ had at least 2 (Table 2). The most frequent concomitant illnesses were cardiovascular diseases $(48 \%)$.

\section{Efficacy}

All 23 patients received 1 or more cycles of regorafenib with schedule 2/1 (median, 5 cycles; range, 2-14 cycles) and were evaluable for response and toxicity. The starting dose is illustrated in Table 3. The mean duration of treatment was 4.1 months (range, 1.1-11.7 months). The mean daily dose was $132.4 \mathrm{mg} \pm 24.7 \mathrm{mg}$ (median, $120 \mathrm{mg}$; range, $80-160 \mathrm{mg}$ ), and the planned dose rate was $82.6 \%$.

\section{Table 2 Patient Comorbidities}

\begin{tabular}{l|r}
\hline Comorbidities & N (\%) \\
Cardiovascular & $11(48)$ \\
\hline Hypertension & $9(39)$ \\
\hline Coronary artery disease & $6(26)$ \\
\hline Arrhythmia & $5(22)$ \\
\hline Diabetes mellitus & $5(22)$ \\
\hline Dyslipidemia & $4(17)$ \\
\hline Respiratory & $7(30)$ \\
\hline Genitourinary & $3(13)$ \\
\hline$>1$ comorbidities & $17(74)$ \\
\hline
\end{tabular}




\section{Regorafenib in Elderly Patients}

\begin{tabular}{|c|c|c|}
\hline & N (\%) & Causes \\
\hline \multicolumn{3}{|l|}{ Starting Dose } \\
\hline $160 \mathrm{mg}$ & $8(35)$ & Fit, $<80$ y \\
\hline $120 \mathrm{mg}$ & $12(52)$ & Vulnerable, or $>1$ comorbidity \\
\hline $80 \mathrm{mg}$ & $3(13)$ & $\geq 80$ y or ECOG PS $=2$ \\
\hline \multicolumn{3}{|l|}{ Escalated dose } \\
\hline From $120 \mathrm{mg}$ to $160 \mathrm{mg}$ & $4(17)$ & After $1,2,2,3$ cycles, respectively \\
\hline From $80 \mathrm{mg}$ to $120 \mathrm{mg}$ & $3(13)$ & After 2, 2, 3 cycles, respectively \\
\hline \multicolumn{3}{|l|}{ Reduced dose } \\
\hline From $160 \mathrm{mg}$ to $120 \mathrm{mg}$ & $3(13)$ & After $1,2,4$ cycles, respectively \\
\hline From $160 \mathrm{mg}$ to $80 \mathrm{mg}$ & $1(4)$ & After 1 cycle \\
\hline From $120 \mathrm{mg}$ to $80 \mathrm{mg}$ & $1(4)$ & After 3 cycles \\
\hline
\end{tabular}

Abbreviation: ECOG PS = Eastern Cooperative Oncology Group performance status.

No patient achieved a CR or PR. Twelve patients (52.2\%) achieved SD, which was the best response to therapy (Table 4). In this regard, computed tomography scans performed at 2 months highlighted relevant tumor necrosis in the liver and/or in the abdominal lymph nodes of 5 of the 12 patients with stable disease. The 2-month DCR was 52.2\% (95\% CI, 31.6\%-72.6\%) (Table 4). The median PFS was 4.8 months (95\% CI, 3.8-6.3 months), and the median OS was 8.9 months (95\% CI, 6.9-10.6 months) (Figure 1). At a median follow-up of 12.3 months (95\% CI, 3.6-15.7 months), a total of 18 patients were deceased.

Regorafenib was discontinued owing to disease progression and treatment-related adverse events in $91 \%$ and $9 \%$ of cases, respectively. Four patients are still on treatment at data cut-off. Seven patients who progressed after regorafenib received the following anti-cancer treatments: oxaliplatin and capecitabine was administered to 3 patients, capecitabine and cetuximab to 3 patients, and capecitabine alone to 1 patient.

\section{Treatment Toxicity}

Adverse events rates are reported in Table 5. The most frequent grade 3 side effects were fatigue, which occurred in 2 (9\%) patients, and hand-foot skin (HFS) reactions, which were reported in 2 (9\%) patients. Among grade 2 or lower adverse events, stomatitis, HFS, and hypertransaminasemia were the most commonly observed. Five patients had grade 1 or 2 cardiac disorders, but no heart failure

\section{Table 4 Results}

\begin{tabular}{l|c} 
Variables & N (\%) \\
Objective response rate & \\
$\quad$ Complete response & 0 \\
\hline Partial response & 0 \\
\hline Stable disease & $12(52)$ \\
\hline$\quad$ Progressive disease & $11(48)$ \\
\hline Two-month disease control rate, \% (95\% Cl, \%) & $52.2(31.6-72.6)$ \\
\hline Progression-free survival, mos $(95 \% \mathrm{Cl}, \mathrm{mos})$ & $4.8(3.8-6.3)$ \\
\hline Overall survival, mos (95\% Cl, mos) & $8.9(6.9-10.6)$ \\
\hline
\end{tabular}

Abbreviation: $\mathrm{Cl}=$ Confidence interval.
Figure 1 Kaplan-Meier Estimates of Overall Survival

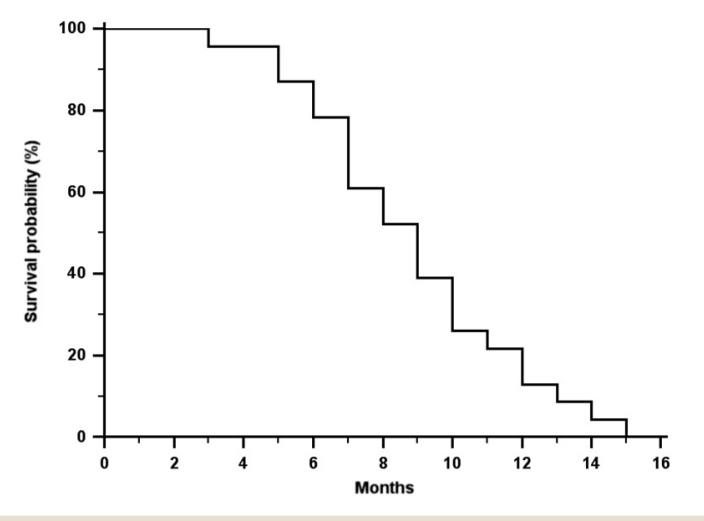

occurred. Hematologic toxicity was mild. No patient required hospitalization because of adverse events. Owing to toxicity, a dose reduction was required in $5(22 \%)$ patients, 4 of whom had started with the conventional dose of $160 \mathrm{mg}$. Regorafenib was re-escalated to $160 \mathrm{mg}$ daily in 4 patients who had started with $120 \mathrm{mg}$. Two (9\%) patients interrupted the treatment: because of persistent HFS after 4 cycles in 1 case and because of continuous HFS and fatigue after 4 cycles in the other (Table 5).

\section{Discussion}

Despite the European Medicine Agency and United States Food and Drug Administration approval of regorafenib as salvage treatment for patients with mCRC who progressed after all available therapies, the not neglectable toxicity profile of the conventional 3/1 schedule as well as the lack of efficacy and safety data on the elderly population limit its use in clinical practice for the older adult. ${ }^{6}$ To our knowledge, the present study is the first suggesting that an

\section{Table 5 Adverse Events}

\begin{tabular}{l|c|c|c} 
Adverse Events & $\begin{array}{c}\text { Grade } \mathbf{3 2 ,} \\
\text { N (\%) }\end{array}$ & $\begin{array}{c}\text { Grade 3, } \\
\text { N (\%) }\end{array}$ & $\begin{array}{c}\text { Grade 4, } \\
\text { N (\%) }\end{array}$ \\
Fatigue & $3(13)$ & $2(9)$ & 0 \\
Hand-foot skin reaction & $4(17)$ & $2(9)$ & 0 \\
Diarrhea & $3(13)$ & $1(4)$ & 0 \\
\hline Hypertension & $3(13)$ & $1(4)$ & 0 \\
\hline Rash or desquamation & $3(13)$ & 0 & 0 \\
Nausea & $2(9)$ & 0 & 0 \\
\hline Vomiting & $1(4)$ & 0 & 0 \\
\hline Stomatitis & $4(17)$ & 0 & 0 \\
Constipation & $3(13)$ & $1(4)$ & 0 \\
\hline Anorexia & $2(9)$ & $1(4)$ & 0 \\
Cardiac disorders & $3(13)$ & 0 & 0 \\
Hypertransaminasemia & $4(17)$ & $1(4)$ & 0 \\
Hyperbilirubinemia & $2(9)$ & 0 & 0 \\
Neutropenia & $2(9)$ & 0 & 0 \\
Anemia & $3(13)$ & 0 & 0 \\
\hline Trombocytopenia & $2(9)$ & 0 & 0 \\
\hline
\end{tabular}


alternative $2 / 1$ schedule of regorafenib is tolerable and efficient for patients aged $\geq 75$ years and screened as non-frail with late-stage mCRC. In fact, despite the limitations of a small observational study, regorafenib seems at least as active in our population as in that of the CORRECT trial. Similar to the latter, in our study there was no $\mathrm{CR}$, and $\mathrm{PR}$ and disease stabilization was the best response to treatment, yet the 2-month DCR was $52.2 \%$, the median PFS was 4.8 months (95\% CI, 3.8-6.3 months), and the median OS was 8.9 months (95\% CI, 6.9-10.6 months) versus $41 \%(P<.0001)$, 1.9 months (95\% CI, 1.6-3.9 months), and 6.4 months (95\% CI, 3.6-11.8 months), respectively, in the CORRECT study. ${ }^{6}$ Furthermore, our findings compare well also with those described by the international phase III trial CONCUR (asian subjects with metastatic COlorectal caNCer treated with regorafenib or placebo after failURe of standard therapy), which compared regorafenib with best supportive care for Asian patients and reported a median OS of 8.8 months (95\% CI, 7.3-9.8 months) in the regorafenibtreated group. $^{13}$ A recent large retrospective Japanese study assessed the efficacy of regorafenib versus the new agent trifluridine/ tipiracil (TFTD) for patients with mCRC who were refractory to standard chemotherapy. ${ }^{14}$ The subgroup analysis by age reported a median OS for the patients $\geq 65$ years old treated with regorafenib of 6.2 months (95\% CI, 4.9-7.4 months), which is comparable to that of the CORRECT trial and slightly shorter than that observed in our analysis. The incidence of discontinuation because of treatment-related toxicities was $24 \%$ in the regorafenib group versus $7 \%$ in the TFTD group, and the authors argued that regorafenib tolerance, unlike TFTD, decreased in elderly patients compared with younger patients. In a population aged 75 years or older (more than one-third were at least 80 years of age), with at least 1 comorbidity ( $74 \%$ with 2$)$, and who progressed after the standard chemotherapy lines for mCRC, further treatment could be questioned as the toxicity can easily outweigh the potential benefit. For this reason, this study used standard, validated tools of geriatric assessment to guarantee that regorafenib would be delivered with a personalized starting dose and only to non-frail patients. As a matter of fact, at baseline, $78 \%$ of patients were considered fit by CGA and, after a median follow-up of 12.3 months (95\% CI, 3.6-15.7 months), 5 patients were still alive and 4 are still on treatment at data cut-off. The use of a 2/1 schedule of administration allowed for shortening the exposure to the drug, and this probably contributed to the good tolerability of treatment with no unexpected severe side effects. This is quite remarkable considering the advanced age of the population, the amount of chemotherapy previously received, and the presence of at least 1 concomitant disease. In particular, $48 \%$ of our population presented with cardiac illnesses at baseline, and recently, a large retrospective study comparing safety of targeted therapies for mCRC between older and younger adults reported more frequent cardiac disorders in the elderly patients treated with bevacizumab, cetuximab, and regorafenib. ${ }^{15}$ In the current study, cardiac disorders were mild, with only 1 case of grade 3 hypertension, and no heart failure occurred. In general, the observed toxicity was milder than in the CORRECT trial. In this respect, except for a similar incidence of grade 3 fatigue ( $9 \%$ ), the rates of nearly all grade 3 adverse events were lower than in the pivotal trial. Consequently, in the CORRECT study, the dose reductions and treatment interruptions rates were considerably higher $(38 \%$ and $61 \%$, respectively) than in our analysis (22\% and 9\%, respectively). Notably, 4 of the 5 patients who required a dose decrease in the present study had started regorafenib at the standard dose, whereas only 1 had a reduced starting dose of $120 \mathrm{mg}$. It should be noted that the starting dose was, in most cases, lower than the standard dose of $160 \mathrm{mg}$, and the $2 / 1$ schedule allowed for a reduced treatment exposure over time. However, the mean daily dose was $132.4 \mathrm{mg}$, and the planned dose rate was $82.6 \%$, which compare well with the mean daily dose of $147.1 \mathrm{mg}$ and the dose intensity of $78.9 \%$ reported in the CORRECT trial.

Additionally, the reported safety profile was comparable to that of the REBECCA (REgorafeniB in mEtastatic Colorectal cancer: a french Compassionate progrAm) study, which analyzed in a real-life setting, the efficacy and toxicity of regorafenib given to patients with mCRC refractory to standard treatments. ${ }^{16}$ Interestingly, almost one-half $(47.6 \%)$ of the 1178 patients enrolled in this study were elderly. However, the side effects required treatment interruptions and dose reductions in $31 \%$ and $43 \%$ of patients, respectively, and thus were not as easily manageable as in our study. ${ }^{16}$ This is probably the result of a combination of factors in our analysis, including the shorter exposure to regorafenib allowed by the modified 2/1 schedule, the accurate selection of non-frail subjects, and the starting dose reductions for the patients who were non-fit or aged $\geq 80$ years. In this regard, as far as we are aware, no data surrounding the use of a modified schedule of regorafenib for mCRC have been previously reported. However, similar experiences have been documented with the multikinase inhibitor sunitinib for the treatment of metastatic renal cell cancer to improve its safety profile. A small study showed a better toxicity profile for sunitinib in a 2-weeks-on/1-week-off regimen compared with the conventional $4 / 2$ schedule, while maintaining the standard dose intensity. ${ }^{17}$ Moreover, a large retrospective analysis reported a better tolerability and no decrease in efficacy for the patients with metastatic renal cell cancer who switched from the standard $4 / 2$ to the modified $2 / 1$ schedule of sunitinib owing to adverse events. ${ }^{18}$

Despite the encouraging results of our analysis, the small size of the population is a limitation that prevents us from drawing general conclusions. Larger randomized trials of comparison between the conventional $3 / 1$ and the alternative $2 / 1$ schedule of administration should be performed in order to confirm our safety and efficacy results. However, these data showed that regorafenib given with a modified 2/1 schedule as last-line treatment for non-frail, 75 years or older patients with mCRC who are refractory to standard chemotherapy is well-tolerated and efficient. Finally, this altered schedule may also be relevant for patients younger than 75 years as the on-label dose and schedule of $160 \mathrm{mg}$ for 3 weeks on, 1 week off is not well-tolerated. Further studies will be required to verify whether the modified $120 \mathrm{mg} 2 / 1$ dose and schedule might be a more tolerated and equally effective regimen for all patients, regardless of age.

\section{Conclusion}

Although careful monitoring of potential side effects is still recommended, this analysis suggests that regorafenib given with a modified $2 / 1$ schedule may be safely proposed for selected elderly patients with mCRC who failed previous standard chemotherapy. 


\section{Regorafenib in Elderly Patients}

\section{Clinical Practice Points}

- In the CORRECT randomized trial, regorafenib was shown to prolong survival of patients with treatment-refractory mCRC. However, there is little data in the literature over the tolerability and efficacy of regorafenib in elderly patients or when administered with a different schedule. Additionally, regorafenib-related adverse events were not neglectable and mostly occurred during cycle 1 to 2 .

- In this prospective study, 23 patients with mCRC who were $\geq$ 75 years old, had progressed after the standard lines of chemotherapy, and were screened as non-frail received regorafenib with a modified schedule consisting of 2 weeks on treatment and 1 week off. More than one-half (52.2\%) of the patients obtained disease stabilization, and both median OS and PFS compared well with those observed in the CORRECT study. Adverse events, in particular grade 3, were uncommon and led to only 5 dose modifications and 2 treatment discontinuations.

- A modified $2 / 1$ schedule of regorafenib combined with an initially personalized starting dose might be safely proposed for selected elderly patients $\geq 75$ years with mCRC.

\section{Disclosure}

The authors have stated that they have no conflicts of interest.

\section{Supplemental Data}

Supplemental data accompanying this article can be found in the online version at https://doi.org/10.1016/j.clcc.2018.02.005.

\section{References}

1. Siegel R, Naishadham D, Jemal A. Cancer statistics, 2013. CA Cancer J Clin 2013; 63:11-30.

2. Aparicio T, Pamoukdjian F, Quero L, et al. Colorectal cancer care in elderly patients: unsolved issues. Dig Liver Dis 2016; 48:1112-8.

3. Gouverneur A, Salvo F, Berdaï D, et al. Inclusion of elderly or frail patients in randomized controlled trials of targeted therapies for the treatment of metastatic colorectal cancer: a systematic review. J Geriatr Oncol 2018; 9:15-23.
4. Schmieder R, Hoffmann J, Becker M, et al. Regorafenib (BAY 73-4506): antitumor and antimetastatic activities in preclinical models of colorectal cancer. Int J Cancer 2014; 135:1487-96.

5. Sartore-Bianchi A, Zeppellini A, Amatu A, Ricotta R, Bencardino K, Siena S. Regorafenib in metastatic colorectal cancer. Expert Rev Anticancer Ther 2014; 14:255-65.

6. Grothey A, Van Cutsem E, Sobrero A, et al, CORRECT Study Group. Regorafenib monotherapy for previously treated metastatic colorectal cancer (CORRECT): an international, multicentre, randomised, placebo-controlled, phase 3 trial. Lancet 2013; 381:303-12.

7. Schmoll HJ, Van Cutsem E, Stein A, et al. ESMO Consensus Guidelines for management of patients with colon and rectal cancer. A personalized approach to clinical decision making. Ann Oncol 2012; 23:2479-516.

8. Wildiers H, Heeren P, Puts M, et al. International Society of Geriatric Oncology consensus on geriatric assessment in older patients with cancer. J Clin Oncol 2014; 32:2595-603.

9. Soubeyran P, Bellera C, Goyard J, et al. Screening for vulnerability in older cancer patients: the ONCODAGE Prospective Multicenter Cohort Study. PLoS One 2014; 9:e115060.

10. Repetto L, Fratino L, Audisio RA, et al. Comprehensive geriatric assessment adds information to Eastern Cooperative Oncology Group performance status in elderly cancer patients: an Italian Group for Geriatric Oncology Study. J Clin Oncol 2002; 20:494-502.

11. Balducci L, Extermann M. Management of cancer in the older person: a practical approach. Oncologist 2000; 5:224-37.

12. Simon R. Optimal two-stage designs for phase II clinical trials. Control Clin Trials 1989; 10:1-10.

13. Li J, Qin S, Xu R, et al, CONCUR Investigators. Regorafenib plus best supportive care versus placebo plus best supportive care in Asian patients with previously treated metastatic colorectal cancer (CONCUR): a randomised, double-blind, placebo-controlled, phase 3 trial. Lancet Oncol 2015; 16:619-29.

14. Moriwaki T, Fukuoka S, Taniguchi $\mathrm{H}$, et al. Propensity score analysis of regorafenib versus trifluridine/tipiracil in patients with metastatic colorectal cancer refractory to standard chemotherapy (REGOTAS): a Japanese Society for Cancer of the Colon and Rectum Multicenter Observational Study. Oncologist 2018; 23:7-15.

15. Gouverneur A, Claraz P, Rousset M, et al. Comparative safety of targeted therapies for metastatic colorectal cancer between elderly and younger patients: a study using the International Pharmacovigilance Database. Target Oncol 2017; 12:805-14.

16. Adenis A, de la Fouchardiere C, Paule B, et al. Survival, safety, and prognostic factors for outcome with regorafenib in patients with metastatic colorectal cancer refractory to standard therapies: results from a multicenter study (REBACCA) nested within a compassionate use program. BMC Cancer 2016; $16: 412$.

17. Makino K, Yoda K, Tomoishi J, Kume H. Efficacy and tolerability of a low-dose, 2-week administration of sunitinib followed by a week rest (2/1 schedule) for metastatic renal cell carcinoma: a single center experience of six cases. BMC Res Notes 2014; 7:872.

18. Bracarda S, Iacovelli R, Boni L, et al, Rainbow Group. Sunitinib administered on 2/1 schedule in patients with metastatic renal cell carcinoma: the RAINBOW analysis. Ann Oncol 2016; 27:366. 